\title{
Linalool Alleviates A $\beta$ 42-Induced Neurodegeneration via Suppressing ROS Production and Inflammation in Fly and Rat Models of Alzheimer's Disease
}

\author{
Chunyu Yuan $\left(\mathbb{D},{ }^{1}\right.$ Myeongcheol Shin $\mathbb{D D}^{1},{ }^{1}$ Youngjae Park $\left(\mathbb{D},{ }^{1}\right.$ Byoungyun Choi $(\mathbb{D}){ }^{1}$ \\ Seokhui Jang $\mathbb{D}^{1}{ }^{1}$ Chaejin Lim $\mathbb{D}^{1},{ }^{1}$ Hye Sup Yun $\mathbb{D}^{1},{ }^{1}$ Im-Soon Lee $\mathbb{D}^{1,2}$ So-Yoon Won $\mathbb{D},{ }^{1,3}$ \\ and Kyoung Sang Cho $\mathbb{D}^{1,2,3}$
}

${ }^{1}$ Department of Biological Sciences, Konkuk University, Seoul 05029, Republic of Korea
${ }^{2}$ CHANS Research Center, Konkuk University, Seoul 05029, Republic of Korea
${ }^{3}$ Korea Hemp Institute, Konkuk University, Seoul 05029, Republic of Korea

Correspondence should be addressed to So-Yoon Won; sywon@konkuk.ac.kr and Kyoung Sang Cho; kscho@konkuk.ac.kr

Received 21 September 2020; Revised 17 February 2021; Accepted 27 February 2021; Published 11 March 2021

Academic Editor: Mingfu Wang

Copyright (C) 2021 Chunyu Yuan et al. This is an open access article distributed under the Creative Commons Attribution License, which permits unrestricted use, distribution, and reproduction in any medium, provided the original work is properly cited.

\begin{abstract}
Terpenes are vital metabolites found in various plants and animals and known to be beneficial in the treatment of various diseases. Previously, our group identified terpenes that increased the survival of Alzheimer's disease (AD) model flies expressing human amyloid $\beta(\mathrm{A} \beta)$ and identified linalool as a neuroprotective terpene against $\mathrm{A} \beta$ toxicity. Linalool is a monoterpene that is commonly present as a constituent in essential oils from aromatic plants and is known to have anti-inflammatory, anticancer, antihyperlipidemia, antibacterial, and neuroprotective properties. Although several studies have shown the beneficial effect of linalool in AD animal models, the mechanisms underlying the beneficial effect of linalool on $\mathrm{AD}$ are yet to be elucidated. In the present study, we showed that linalool intake increased the survival of the AD model flies during development in a dosedependent manner, while the survival of wild-type flies was not affected even at high linalool concentrations. Linalool also decreases $\mathrm{A} \beta$-induced apoptosis in eye discs as well as the larval brain. Moreover, linalool intake was found to reduce neurodegeneration in the brain of adult $\mathrm{AD}$ model flies. However, linalool did not affect the total amount of $\mathrm{A} \beta 42$ protein or $\mathrm{A} \beta 42$ aggregation. Rather, linalool decreased $\mathrm{A} \beta$-induced ROS levels, oxidative stress, and inflammatory response in the brains of $\mathrm{AD}$ model flies. Furthermore, linalool attenuated the induction of oxidative stress and gliosis by $\mathrm{A} \beta_{1-42}$ treatment in the rat hippocampus. Taken together, our data suggest that linalool exerts its beneficial effects on $\mathrm{AD}$ by reducing $\mathrm{A} \beta 42$-induced oxidative stress and inflammatory reactions.
\end{abstract}

\section{Introduction}

Alzheimer's disease (AD) refers to a neurodegenerative condition, which is recognized as the most common cause of dementia [1]. The hallmarks of $\mathrm{AD}$ are abnormal aggregation of amyloid $\beta(\mathrm{A} \beta)$ and tau protein, and the accumulation of the pathological forms of these proteins has been considered to be a cause of $\mathrm{AD}$ [2]. In particular, the accumulated $\mathrm{A} \beta$ forms toxic oligomers, which subsequently induce various pathophysiological events such as inflammation, reactive oxygen species (ROS) generation, and neuronal death during the progression of $\mathrm{AD}$ [3-6].
Currently, four drugs, including donepezil, galantamine, rivastigmine, and memantine, have been approved by the U.S. Food and Drug Administration for the treatment of $\mathrm{AD}$ [7]. However, these treatments are used as temporary memory enhancers to relieve symptoms rather than to modify disease progression. In addition, these treatments are associated with adverse effects related to the gastrointestinal tract and cardiovascular system [8, 9]. Therefore, studies on the neuroprotective effects of natural compounds that do not exhibit any pronounced toxicity are being conducted to replace the current treatments for $\mathrm{AD}$. 
Terpenes are natural compounds that have a potential to treat $\mathrm{AD}$. They are volatile organic compounds that contain hydrocarbons with 5 carbon atoms as their building blocks. Terpenes are produced by various organisms, especially plants $[10,11]$. Previous studies have shown that many terpenes have anti-inflammatory, antitumor, or neuroprotective properties [12]. Based on that, our prior screening identified terpenes that exhibit neuroprotective effects against $\mathrm{A} \beta$ cytotoxicity using a Drosophila AD model and identified six terpenes, namely, $\rho$-cymene, limonene $(+)$, limonene $(-)$, linalool, $\alpha$-pinene $(+)$, and $\beta$-pinene $(-)$, as neuroprotective terpenes that can effectively suppress the $\mathrm{A} \beta$ phenotype in AD flies [13].

Linalool is a monoterpene that is commonly present as a constituent in essential oils from aromatic plants. Linalool is known to have anticancer, antihyperlipidemia, antibacterial, and neuroprotective properties and has a wide range of biological activities, including antioxidant and antiinflammatory effects $[14,15]$. It is a colorless and volatile monoterpene found in essential oils from more than 200 plants [16, 17]. Several studies have shown that linalool exerts a neuroprotective effect by inhibiting inflammation in vitro and in vivo [18-20]. Linalool also protects neurons by decreasing ROS levels in patients with carpal tunnel syndrome, which is a ROS-induced peripheral neuropathy [21]. Importantly, the safety of linalool is attested by the fact that it has been approved as a food flavoring by the European Commission [22]. Therefore, linalool is a promising natural compound for treating diseases related to ROS and inflammation $[14,15]$.

The beneficial effects of linalool on neurodegeneration were reported in two $\mathrm{AD}$ mouse models. Linalool reduced $\mathrm{A} \beta$ aggregation in a transgenic $\mathrm{AD}$ mouse model and reversed $A \beta$-induced memory loss in both transgenic and $\mathrm{A} \beta$-injected $\mathrm{AD}$ mouse models [23, 24]. However, the detailed molecular mechanisms by which linalool protects the neurons from $\mathrm{A} \beta$ toxicity are not fully understood. Therefore, the use of linalool as a treatment for AD needs to be studied in many respects.

In the present study, we showed that linalool intake suppressed $\mathrm{A} \beta$-induced neuronal cell death without affecting $\mathrm{A} \beta$ aggregation in the brains of flies expressing human $A \beta$. Linalool ameliorated AD-like phenotypes, including neurodegeneration, through its anti-inflammatory and antioxidative properties in fly and rat $\mathrm{AD}$ models. These results suggest that linalool is a potential therapeutic agent for $\mathrm{AD}$ treatment.

\section{Materials and Methods}

2.1. Drosophila Strains. The $w^{1118}$, glass multimer reporterGAL4 (GMR-GAL4; eye driver), embryonic lethal abnormal vision-GAL4 (elav-GAL4; pan-neuronal driver), and Drosomycin-tagged green fluorescent protein (Drs-GFP) strains were obtained from the Bloomington Drosophila Stock Center (Bloomington, IN, USA). The UAS-A $\beta 42^{2 \mathrm{X}}$ strain was a gift from Dr. Fernandez-Funez (University of Florida, USA). The genotypes of the flies were elav $>A \beta 42^{2 \mathrm{X}}$ $\left(\right.$ elav-GAL4/+; UAS-A $\beta 42^{2 \mathrm{X} /+)}$ and $G M R>A \beta 42^{2 \mathrm{X}}(G M R$ -

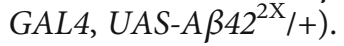

2.2. Survival Assay. In order to determine the survival rate of Drosophila, 250 Drosophila embryos were collected on grape juice agar plates. Every 50 embryos were transferred into a vertical plastic vial and maintained at $25^{\circ} \mathrm{C}$ and $60 \%$ humidity. The survival rate of the female adults was estimated. DMSO-fed $w^{1118}$ and DMSO-fed elav $>A \beta 42^{2 \mathrm{X}}$ were used as controls. This experiment was repeated three times (total of 750 embryos per group were used).

2.3. Acridine Orange Staining. Acridine orange (AO) staining was performed to detect cell death. As previously described [13], the brain or eye discs of L3 larvae were dissected in phosphate buffer saline (PBS, pH7.8, Bio Basic, Seoul, Republic of Korea) and incubated with $1.6 \times 10^{-6} \mathrm{M} \mathrm{AO}$ (Sigma Aldrich, St. Louis, USA) for $5 \mathrm{~min}$ at room temperature. The samples were washed twice with PBS for $5 \mathrm{~min}$ each. The cells were examined under an Axiophot 2 fluorescence microscope (Carl Zeiss, Oberkochen, Germany), and then the number of stained cells was counted.

2.4. Brain Section. To measure neuronal loss, the fly heads were sectioned as previously reported [25]. Fly heads were fixed in Carnoy solution (60\% ethanol, 30\% chloroform, and $10 \%$ glacial acetic acid) at $4{ }^{\circ} \mathrm{C}$ for $4 \mathrm{~d}$ and embedded in paraffin. The embedded heads were sectioned ( $5 \mu \mathrm{m}$ thick sections) and stained with hematoxylin and eosin. The stained samples were examined under a light microscope.

2.5. Immunohistochemical Analysis of Drosophila Brain. Fly brains were fixed in $4 \%$ paraformaldehyde (PFA) prepared in PBST (PBS $+0.5 \%$ Triton $\mathrm{X}-100)$ for $3 \mathrm{~h}$ at room temperature. The samples were washed thrice with PBST for $10 \mathrm{~min}$ and incubated in blocking solution ( $2 \%$ normal goat serum $+2 \%$ bovine serum albumin (BSA) $+0.5 \%$ Triton $\mathrm{X}-100$ ) for $3 \mathrm{~h}$. Then, the samples were incubated with anti-A $\beta 42$ antibody prepared in the blocking solution for $48 \mathrm{~h}$ at $4^{\circ} \mathrm{C}$ (blocking solution 1:200; Santa Cruz, CA, USA). Alexa Fluor 555 anti-rabbit antibody (PBST 1:200; Cell Signaling Technology, Danvers, USA) was used as the secondary antibody.

2.6. Measurement of ROS Levels. The ROS levels in the eye discs were measured using dihydroethidium (DHE; Invitrogen molecular probe, USA). As previously described [13], the eye discs of L3 larvae were dissected and incubated with Schneider's medium containing $40 \mu \mathrm{M}$ DHE for $5 \mathrm{~min}$ and then washed twice in Schneider's medium for $5 \mathrm{~min}$ each time. The samples were observed under an Axiophot 2 fluorescence microscope (Carl Zeiss).

2.7. Measurement of Nitric Oxide Levels. As previously described [13], the heads of 3- to 5-day-old flies $(n=15)$ were resuspended in $10 \times$ PBS on ice. The samples were ground and centrifuged at $10,000 \times g$ for $10 \mathrm{~min}$ at $4^{\circ} \mathrm{C}$. The supernatants were mixed with Greiss reagent at $1: 1$ ratio (SigmaAldrich) and incubated for $15 \mathrm{~min}$ at $25^{\circ} \mathrm{C}$. Nitrite oxide (NO) levels were measured at $550 \mathrm{~nm}$ using a spectrophotometer. 
2.8. Measurement of Inflammation after Bacterial Infection. The DH5 $\alpha$ strain of Escherichia coli (Dongin Biotech, Republic of Korea) was cultured in Luria Bertani medium at $37^{\circ} \mathrm{C}$ for $16-20 \mathrm{~h}$ and concentrated by centrifugation. Septic injury was induced by pricking the thorax of 3- to 5-day-old DrsGFP adult females with a thin needle dipped into a concentrated bacterial pellet $\left(4.4 \times 10^{10} \mathrm{cfu} / \mathrm{ml}\right)[13]$.

2.9. Animals. All experimental procedures were in accordance with the guidelines of the Laboratory Animal Manual of the National Institutes of Health Guide to the Care and Use of Animals, which were approved by the Ethics Review Committee of Konkuk University for Animal Experiments (approval number: KU20170). Sprague-Dawley rats, 260 to $280 \mathrm{~g}$ at the time of surgery, were housed two to three per cage with ad libitum access to water and food during a 12hour light/dark cycle.

2.10. Preparation of $A \beta$ Peptide. $\mathrm{A} \beta_{1-42}$ (Invitrogen, Camarillo, CA) were prepared as described previously [26] by dissolving the peptide in a freshly prepared $35 \%$ acetonitrile solution with dilution to a final concentration of $0.5 \mathrm{mM}$ in PBS ( $\mathrm{pH}$ 7.4). The $\mathrm{A} \beta_{1-42}$ solution was incubated for $24 \mathrm{~h}$ at $37^{\circ} \mathrm{C}$ to prepare fibrillary aggregates. After incubation, $\mathrm{A} \beta_{1-}$ ${ }_{42}$ were stored at $-20^{\circ} \mathrm{C}$ until use.

2.11. Stereotaxic Surgery. Rats were anesthetized and positioned in a stereotaxic apparatus (David Kopf Instruments, Tujunga, CA). A midline sagittal incision was made in the scalp, and holes were drilled in the skull over the dorsal hippocampus using the following coordinates: $3.6 \mathrm{~mm}$ posterior to the bregma and $2.0 \mathrm{~mm}$ lateral to the midline for intrahippocampal injections according to the atlas of Paxinos and Watson [27]. The hole of the tip was directed down to $2.6 \mathrm{~mm}$ beneath the surface of the brain for the hippocampus. All injections were made using a Hamilton syringe equipped with a $30 \mathrm{~S}$ gauge beveled needle and attached to a syringe pump (KD Scientific, New Hope, PA). Infusions were made at a rate of $0.2 \mu \mathrm{l} / \mathrm{min}$ for $\mathrm{A} \beta_{1-42}(1 \mathrm{nmol}$ in $2 \mu \mathrm{l})$. After injection, the needle was left in place for an additional $5 \mathrm{~min}$ before being slowly retracted.

2.12. Linalool Injection. Linalool was administered intraperitoneally (i.p.) once a day for 7 days before surgery and 14 days after surgery. Linalool was dissolved in DMSO (vehicle) and injected at doses of 50 and $100 \mathrm{mg} / \mathrm{kg}$. The control groups received the same volume of vehicle for 3 weeks.

2.13. Rat Brain Tissue Preparation and Immunostaining. Rats were anesthetized at the indicated time points after injection and were perfused transcardially with a $0.9 \%$ saline solution containing $0.5 \%$ sodium nitrate and heparin $(10 \mathrm{U} / \mathrm{ml})$, which was followed by fresh cold 4\% PFA fixative ( $\mathrm{pH}$ 7.4). Brains were removed from the skull, postfixed in 4\% PFA for $24 \mathrm{~h}$ at $4^{\circ} \mathrm{C}$, and then placed in a $30 \%$ sucrose solution until they sank. Brains were frozen sectioned using a sliding microtome into $35 \mu \mathrm{m}$ coronal sections after they were embedded in optimum cutting temperature compound (Surgipath), and they were then collected into six separate series. Sections were stored free-floating in cryopreservative medium at $-20^{\circ} \mathrm{C}$. The tissue sections were washed in cold PBS for $15 \mathrm{~min}$ and incubated with a universal blocking solution in PBS (0.3\% Triton X-100, 1\% BSA, 0.05\% Tween 20, $0.1 \%$ cold fish gelatin, and $0.05 \%$ sodium azide) for $1 \mathrm{~h}$ at room temperature. Brain sections were rinsed in PBS then incubated with the following primary antibodies: antiglial fibrillary acidic protein (GFAP, 1:500, mouse, Sigma Aldrich) for astrocytes and anti-4-hydroxynonenal (4-HNE, $1: 300$, mouse, abcam, Cambridge, UK) for lipid peroxidation. For fluorescence microscopy, the samples were incubated with Alexa 594-conjugated secondary antibodies (Cell Signaling Technology). For light microscopy, brain tissues were incubated with a biotin-conjugated secondary antibody followed by streptavidin-conjugated HRP (Vectastain ABC kit, Vector Laboratories). Immunostaining was visualized by incubating the samples in a $0.1 \mathrm{M}-\mathrm{PB}$ solution containing $0.05 \%$ diaminobenzidine- $\mathrm{HCl}$ and $0.003 \%$ hydrogen peroxide.

2.14. Statistical Analysis. Data were quantitatively analyzed for significance using either Student's $t$-test (two-tailed) or one-way ANOVA followed by Tukey-Kramer multiple comparison test. Student's $t$-test was used for comparisons between two groups. GraphPad Prism 8.0 (GraphPad Software Inc., USA) was used for performing statistical analyses. $P$ values $<0.05$ indicated a significant difference.

\section{Results}

3.1. Linalool Intake Increases Survival during the Development of the Drosophila AD Model. It is known that the Drosophila AD model used in this study shows decreased survival compared to the wild-type Drosophila during development [13]. To confirm the effect of linalool on $\mathrm{AD}$, we used the Drosophila AD model (elav-GAL4>UAS-A $\left.342^{2 \mathrm{X}}\right)$ that expresses $A \beta 42$ in the neurons. Donepezil (Done), a current $\mathrm{AD}$ treatment, was used as a positive control (Figure 1(a)). As expected, donepezil increased the survival of $A \beta 42-$ expressing flies during development (Figure 1(a)). Linalool also showed protective effects against $\mathrm{A} \beta 42$ toxicity in a dose-dependent manner. More importantly, linalool did not affect the survival of wild-type flies at the concentrations used in AD model flies (Figure 1(b)). These results demonstrate that linalool has a protective effect against $\mathrm{A} \beta$ toxicity and has no apparent harmful effects even at high concentrations.

3.2. Linalool Intake Suppressed A $\beta 42-I n d u c e d$ Cell Death. As linalool reduced $\mathrm{A} \beta$ toxicity during development, we investigated whether linalool intake suppressed $A \beta 42$-induced cell death in the developing eyes and the brain using AO staining, which detects dead cells. Similar to a previous report [13], ectopic expression of human $A \beta 42$ in the developing eyes using $G M R$-GAL4 driver $\left(G M R>A \beta 42^{2 \mathrm{X}}\right)$ strongly induced cell death (Figures 2(a) and 2(b)). Linalool and donepezil significantly reduced $\mathrm{A} \beta 42$-induced cell death in the developing eye discs (Figures 2(a) and 2(b)). Consistently, linalool and donepezil treatment also suppressed $\mathrm{A} \beta 42$-induced cell death in the larval brain (Figures 2(c) and 2(d)). We also examined whether linalool intake suppresses 


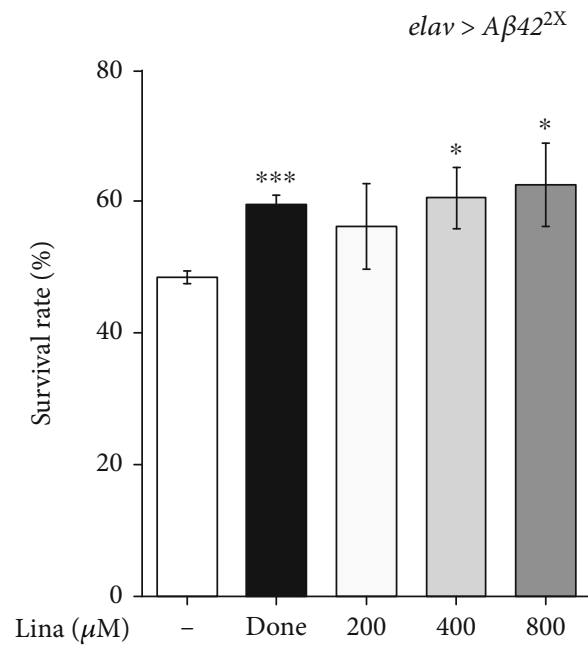

(a)

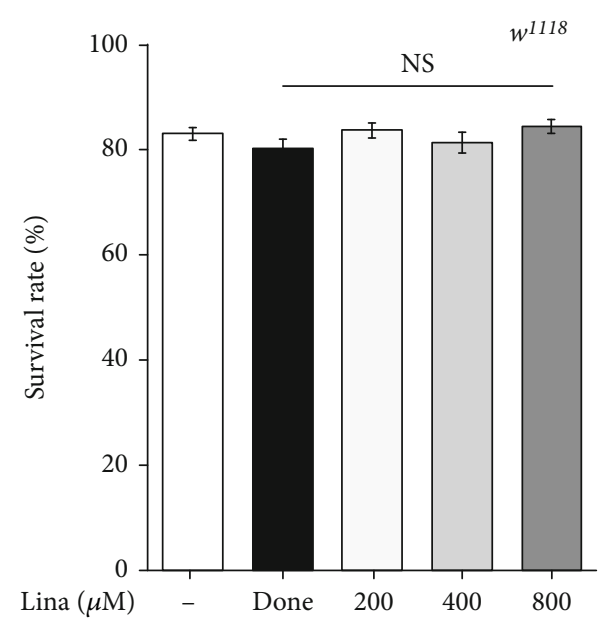

(b)

FIgURE 1: Linalool intake increases survival of the Drosophila AD model during development. The graphs show the effect of linalool on the survival of Drosophila expressing $A \beta 42$ (elav $\left.>A \beta 42^{2 \mathrm{X}}\right)(\mathrm{a})$ and $w^{1118}$ (b) during development $(n=750)$. Flies fed with donepezil (Done) were used as positive control. All data in the graphs are expressed as mean \pm standard error of mean (SEM). ${ }^{* * *} P<0.001,{ }^{*} P<0.05$ vs. DMSO-fed elav $>A \beta 42^{2 \mathrm{X}}$ (a) or DMSO-fed $w^{1118}$ (b); Student $t$-test. Done: $0.1 \mu \mathrm{g} / \mathrm{ml}$ donepezil; Lina: linalool; NS: not significant.

neurodegeneration in the brains of $A \beta 42$-expressing flies. Neurodegeneration leads to vacuolation in the brains of $\mathrm{AD}$ model flies [28], which is not observed in the brains of wild-type flies (Figure 2(e)). The vacuole area in the brains of flies fed with linalool was significantly decreased compared to that in the brains of control (DMSO-fed ela$v>A \beta 42^{2 \mathrm{X}}$ ) flies (Figures $2(\mathrm{e})$ and $2(\mathrm{f})$ ), demonstrating that feeding with linalool reduced neurodegeneration. Collectively, our results suggest that linalool protects neurons from neurodegeneration by inhibiting neuronal cell death in $\mathrm{AD}$ model flies.

\subsection{Linalool Did Not Affect A $\beta 42$ Protein Levels or} Aggregation. As A $\beta 42$ aggregation and deposition are closely related to neuronal cell death, we investigated whether the inhibitory effect of linalool on cell death is due to a decrease in the amount of $\mathrm{A} \beta 42$ deposition or aggregation. Therefore, we compared the amount of $A \beta 42$ protein accumulation in the brains of $\mathrm{AD}$ flies fed with linalool to that in brains of DMSO-fed control flies. The accumulated A $\beta 42$ was visualized using immunohistochemistry. As shown in Figures 3(a) and 3(b), linalool intake did not alter $A \beta 42$ levels in the brains of $\mathrm{AD}$ flies. We also examined the levels of $\mathrm{A} \beta 42$ aggregates by thioflavin $S$ staining, which stains oligomeric fibrils or plaques of A $\beta 42$ [29]. Similar to accumulated $\mathrm{A} \beta 42$ levels, aggregation of $\mathrm{A} \beta 42$ was not altered by linalool (Figures 3(c) and 3(d)). These results indicate that the neuroprotective effect of linalool is not achieved by inhibiting $\mathrm{A} \beta 42$ accumulation or aggregation.

\subsection{Linalool Decreased A $\beta 42-I n d u c e d$ ROS Generation.} Given that linalool has an antioxidant effect [12], and that ROS is an important mediator of A $\beta 42$ toxicity [30], ROS was measured by DHE staining to determine whether linalool prevents ROS production in $\mathrm{AD}$ flies through its antioxidant activity. Similar to a previous report [13], ectopic expression of $A \beta 42$ in the developing eyes $\left(G M R>A \beta 42^{2 \mathrm{X}}\right)$ clearly induced ROS levels, as detected by DHE staining, while DHE-positive signals were not observed in control flies without A 342 (GMR-GAL4) (Figure 4). Linalool and donepezil significantly decreased ROS levels (Figure 4), suggesting that the protective effects of linalool against $A \beta 42$ toxicity are related to its antioxidant effect, at least in part.

3.5. Linalool Decreased Inflammation Induced by $A \beta 42$ and Bacterial Infection. Previous studies have shown that neuroinflammation plays a key role in the pathology of $\mathrm{AD}$ [5]. As linalool has been reported to exert anti-inflammatory effects $[31,32]$, we investigated whether the beneficial effect of linalool is owing to its suppressive function in inflammation. First, to examine whether linalool can reduce inflammation in Drosophila, we used a microbial infection model, in which inflammatory response can be measured by an antimicrobial peptide reporter system after septic injury that infects the microorganism. In this system, the levels of inflammatory response can be measured based on the expression levels of GFP-labeled Drosomycin (DrsGFP) in Drosophila. As expected, GFP levels increased in the thorax of Drosophila following DH5 $\alpha$ infection when compared to those in the thorax of sucrose-injected control Drosophila (Figures 5(a) and 5(b)). Interestingly, linalool intake significantly reduced the expression of $\mathrm{DH} 5 \alpha$ infection-induced Drs-GFP (Figures 5(a) and 5(b)). Then, we assessed whether linalool intake reduced the inflammation caused by $\mathrm{A} \beta 42$ by measuring NO levels in the fly brains. Similar to a previous report [13], $A \beta 42$ expression in neurons increased NO levels in the brain of flies (Figure 5(c)). Moreover, linalool decreased NO levels in the $\mathrm{AD}$ flies to the levels of the control flies (Figure 5(c)). These results indicate that linalool suppresses inflammation in $\mathrm{AD}$ flies through its anti-inflammatory effect. 


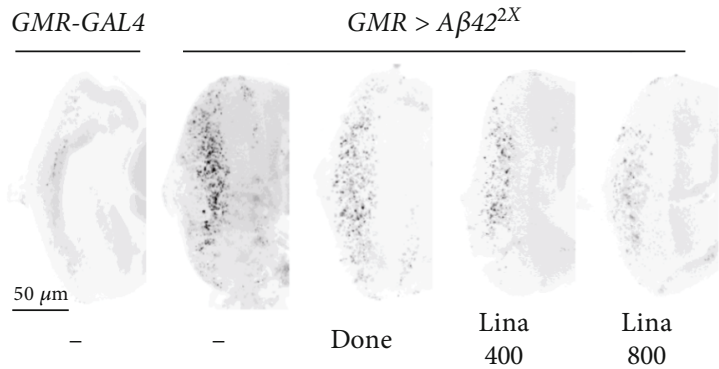

(a)

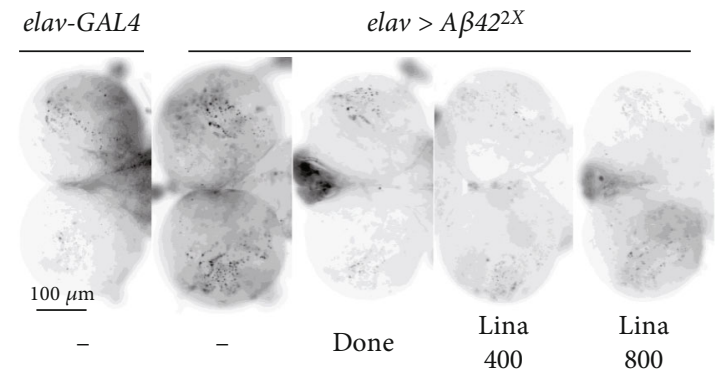

(c)

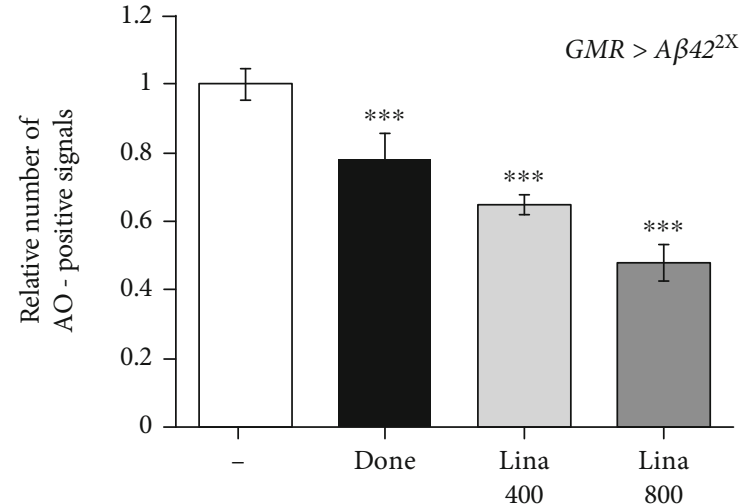

(b)

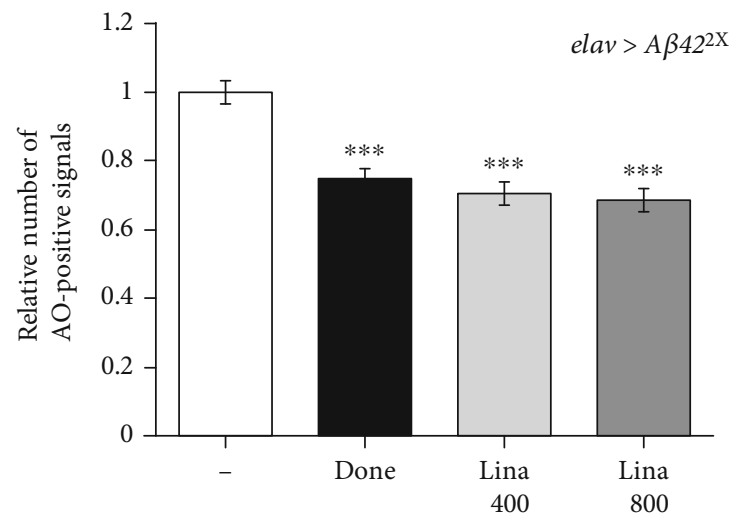

(d)

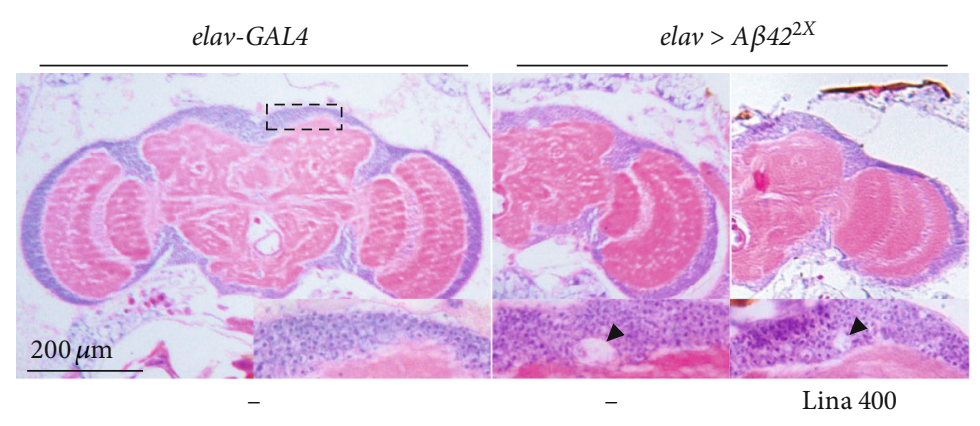

(e)

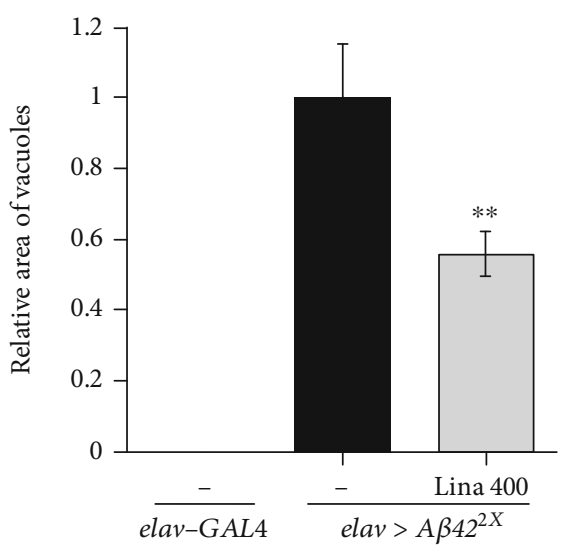

(f)

Figure 2: Linalool intake suppresses A $\beta 42$-induced cell death and neurodegeneration. Linalool inhibited cell death in the Drosophila eye discs $(\mathrm{a}, \mathrm{b})$ and the larval brains (c, d) expressing $A \beta 42$. ( $\mathrm{a}, \mathrm{c})$ Representative images of acridine orange- (AO-) stained larval eye discs (a) and brains (c) of indicated groups. (b, d) The graphs show the relative number of AO-positive signals in the larval eye discs and brains of indicated groups $(n \geq 20)$. All data in the graphs are expressed as mean \pm SEM. ${ }^{* * *} P<0.001$ vs. DMSO-fed elav $>A \beta 42^{2 \mathrm{X}}$ (b) or DMSO-fed $G M R>A \beta 42^{2 \mathrm{X}}(\mathrm{d})$; Student's $t$-test. (e, f) Linalool inhibited neurodegeneration in the brains expressing $A \beta 42$. (e) Representative optical microscopic images of the adult brains of control (elav-GAL4) and the $\mathrm{AD}$ model (elav $>A \beta 42^{2 \mathrm{X}}$ ). The dotted box is the region in which vacuoles were observed. Insets are magnified views of dorsomedial region of the brain in which vacuoles appeared. Arrowheads indicate vacuoles caused by neurodegeneration. (f) The graph shows the relative size of vacuole areas $(n \geq 7)$. All data in the graphs are expressed as mean \pm SEM. ${ }^{* *} P<0.01$ vs. DMSO-fed elav $>A \beta 42^{2 \mathrm{X}}$ (f); Tukey-Kramer test. Done: $0.1 \mu \mathrm{g} / \mathrm{ml}$ donepezil; Lina 400 : $400 \mu \mathrm{M}$ linalool; Lina 800: $800 \mu \mathrm{M}$ linalool.

3.6. Linalool Reduced $A \beta_{1-42}$-Induced Oxidative Stress and Gliosis in the Rat Hippocampus. To determine whether these finding extend to a mammalian model of $\mathrm{AD}$, the rat unilateral $\mathrm{A} \beta_{1-42}$ lesion model of $\mathrm{AD}$ was used [26]. $\mathrm{A} \beta_{1-42}$ and vehicle were stereotaxically injected into the hippocampus. Linalool was administered intraperitoneally once a day for 7 days before $\mathrm{A} \beta_{1-42}$ injection and for 14 days after $\mathrm{A} \beta_{1-42}$ injection (Figure 6(a)). To investigate effects of linalool on 


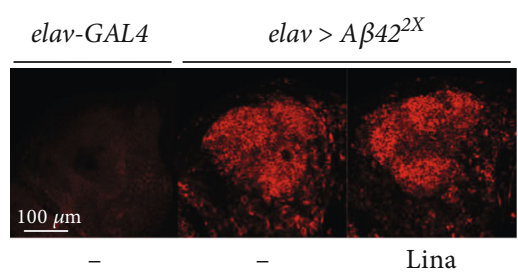

(a)

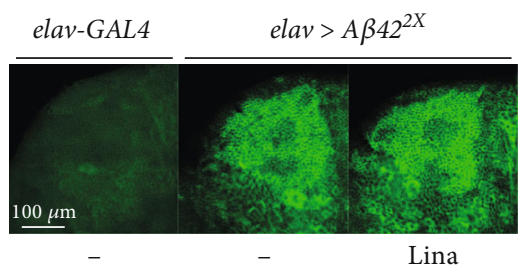

(c)

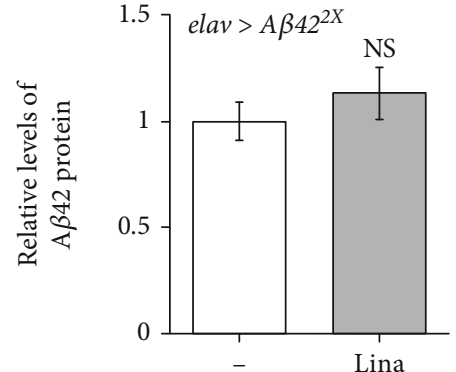

(b)

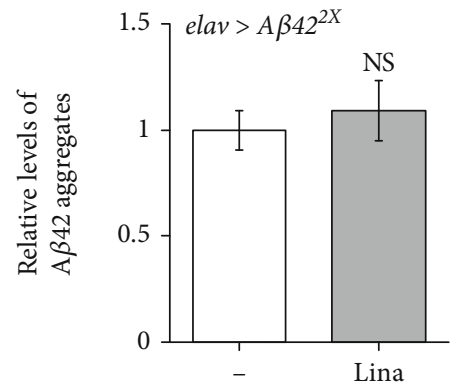

(d)

FIgURE 3: Linalool intake does not affect $\mathrm{A} \beta 42$ peptide accumulation and aggregation. The levels of $\mathrm{A} \beta 42$ peptide $(\mathrm{a}, \mathrm{b})$ and aggregation (c, d) in the Drosophila brains expressing $A \beta 42$ (elav>A $\beta 42^{2 \mathrm{X}}$ ) were not altered by linalool intake. (a, c) Representative confocal images of the Kenyon cell body region in the adult fly brains. The $\mathrm{A} \beta 42$ protein accumulation (a) and aggregation (c) in the brains of flies fed with linalool (elav $>A \beta 42^{2 \mathrm{X}}$, Lina) were compared to those in the brains of control (elav>A $\beta 42^{2 \mathrm{X}}$, DMSO) flies. Adult brains were stained with anti-A $\beta 42$ antibody (a) or thioflavin $\mathrm{S}(\mathrm{c})$. (b, d) The graphs show the relative intensity of $\mathrm{A} \beta 42$-antibody staining (b) and thioflavin $\mathrm{S}$ staining (d) $(n \geq 20)$. All data in the graphs are expressed as mean \pm SEM; Student's $t$-test. Lina: $400 \mu$ M linalool; NS: not significant.

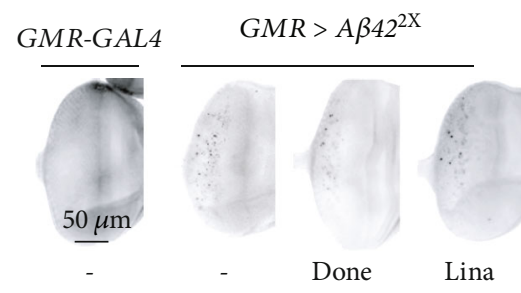

(a)

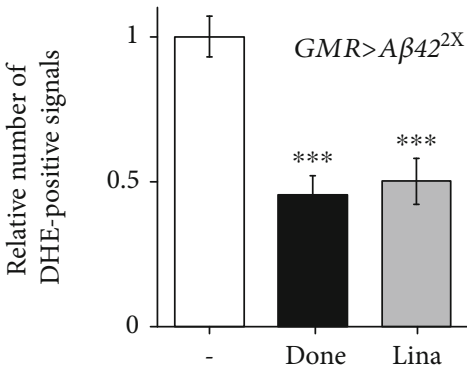

(b)

FIgURE 4: Linalool intake decreases ROS levels in the eye discs expressing $A \beta 42$. (a) Representative DHE-staining images of larval eye discs expressing $A \beta 42\left(G M R>A \beta 42^{2 \mathrm{X}}\right)$. DHE-positive signals in the eye discs of linalool-fed flies were compared to those in the eye discs of control $\left(G M R>A \beta 42^{2 \mathrm{X}}\right.$, DMSO fed) flies. (b) The graph shows the relative number of DHE-positive signals $(n \geq 10)$. All data in the graph are expressed as mean \pm SEM. ${ }^{* * *} P<0.001$ vs. DMSO-fed $G M R>A \beta 42^{2 X}$ (b); Student's $t$-test. Done: $0.1 \mu \mathrm{g} / \mathrm{ml}$ donepezil; Lina: $400 \mu \mathrm{M}$ linalool.

$\mathrm{A} \beta_{1-42}$-induced oxidative damage, we performed immunostaining with an anti-4-HNE antibody in the hippocampal tissues. Immunohistochemical analysis showed that 4-HNE levels were significantly increased in the CA1 layer of the hippocampus of $A \beta_{1-42}$-treated rats compared to vehicle-treated rats (Figures 6(b) and 6(c)). This $A \beta_{1-42}$-induced increase of 4-HNE levels was significantly attenuated at 21 days after linalool treatment (50 and $100 \mathrm{mg} / \mathrm{kg} /$ day) (Figures 6(b) and $6(c))$. Next, we used a GFAP antibody to examine glial reactivity in the hippocampus of $\mathrm{A} \beta_{1-42}$-induced rats. Increased GFAP immunostaining was detected in the hippocampus of $\mathrm{A} \beta_{1-42}$-injected rats as compared to the control. Hypertro- phic astrocytes were apparent in the hippocampus of the $\mathrm{A} \beta_{1-42}$-injected rats, while linalool treatment (50 and $100 \mathrm{mg} / \mathrm{kg} /$ day) reduced the number of GFAP+ immunoreactive astrocytes (Figures 6(d) and 6(e)). Taken together, these results provide evidence that linalool can prevent $\mathrm{A} \beta_{1-42}$-induced oxidative stress and gliosis in the hippocampus of rats.

\section{Discussion}

In our previous study, we discovered linalool as one of the major monoterpenes secreted in forests and that it has the 


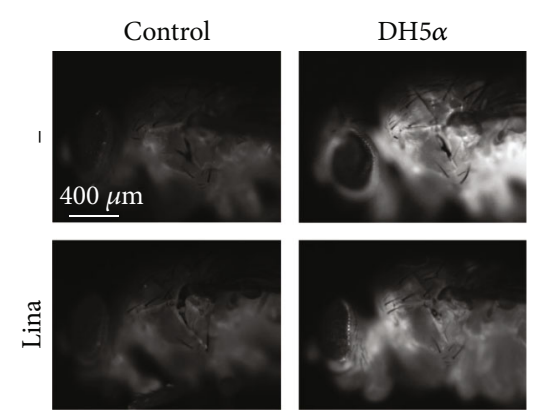

(a)

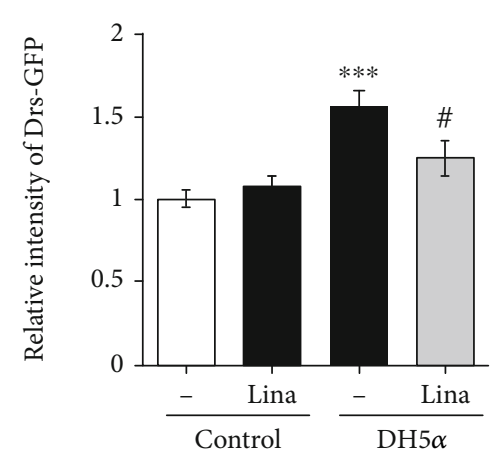

(b)

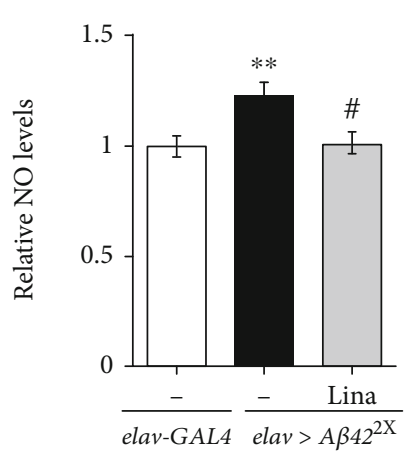

(c)

Figure 5: Linalool intake reduces inflammation induced by bacterial infection or $A \beta 42$ expression. (a) Fluorescent microscopic images of linalool- or DMSO-fed Drs-GFP flies that express GFP controlled by the Drs promoter. Sucrose is used for a negative control. (b) The graph shows the relative levels of GFP in the thorax of flies injected with sucrose or DH5 $(n \geq 10)$. (c) The effect of linalool intake on the elevated NO levels in $A \beta 42$-expressing fly heads. The graph shows relative NO levels in the heads of adult flies $(n \geq 9)$. NO levels in the heads of flies expressing $A \beta 42$ (elav $>A \beta 42^{2 \mathrm{X}}$ ) were compared to those in the heads of control (elav-GAL4) flies. All data in the graphs are expressed as mean \pm SEM. ${ }^{* * *} P<0.001,{ }^{* *} P<0.01$ vs. DMSO-fed elav-GAL4 (b) or DMSO-fed Drs-GFP injected with sucrose (c), ${ }^{\#} P<0.05$ vs. DMSO-fed elav $>A \beta 42^{2 \mathrm{X}}$ (b) or DMSO-fed Drs-GFP injected with DH5 $\alpha$ (c); Tukey-Kramer test. Lina: $400 \mu \mathrm{M}$ linalool; DrsGFP: GFP-tagged Drosomycin.

potential to alleviate the cytotoxicity of $\mathrm{A} \beta[13]$. Based on that, in this study, we investigated how linalool exhibits neuroprotective effects using Drosophila models that express human $\mathrm{A} \beta$ in neurons or developing eyes as well as an $\mathrm{A} \beta_{1-42}$-injected rat model. As a result, we found that linalool suppressed ROS production and inflammatory response induced by $\mathrm{A} \beta$ expression and reduced neurodegeneration. These results are consistent with the results of previous studies showing that linalool has antioxidant and anti-inflammatory properties in mouse models and in vitro assay $[17,33]$, which suggests that these properties are the main mechanisms underlying the neuroprotective effect of linalool.

Several previous studies have reported the neuroprotective effect of linalool $[20,34]$. In fact, linalool mitigates cognitive impairment and brain damage caused by $\mathrm{A} \beta$ in a transgenic AD mouse model [23]. Moreover, linalool decreases the amount of $\mathrm{A} \beta$ in the brains of mice, but it is unclear whether linalool decreases $\mathrm{A} \beta$ production or increases $A \beta$ clearance [23]. The amount of $A \beta$ accumulation is determined by the sum of $\mathrm{A} \beta$ production and $\mathrm{A} \beta$ clearance in the triple transgenic $\mathrm{AD}$ mouse model expressing $\mathrm{PS}_{1 \mathrm{M} 146 \mathrm{~V}}, \operatorname{tau}_{\mathrm{P} 301 \mathrm{~L}}$, and $\mathrm{APP}_{\mathrm{swe}}$. However, the Drosophila model used in the present study does not undergo $\beta$-amyloidosis and directly expresses A $\beta 42$; therefore, in our model, we only observed whether linalool affects $A \beta$ clearance. As linalool did not affect the accumulation and aggregation of $\mathrm{A} \beta 42$ in our model, we believe that linalool does not influence $\mathrm{A} \beta$ clearance. Herein, linalool seems to inhibit $\mathrm{A} \beta$ production rather than its clearance. Further studies are needed to determine how linalool inhibits $\mathrm{A} \beta$ production in the future.

In addition, linalool inhibits not only $\mathrm{A} \beta$ accumulation but also free radical formation and inflammatory reaction induced by $\mathrm{A} \beta$ [23]. However, from these results alone, it is unknown whether linalool inhibits $\mathrm{A} \beta$ formation and consequently inhibits free radical formation and inflammatory responses or whether it inhibits $\mathrm{A} \beta$ formation while simultaneously inhibiting free radical formation and inflammatory responses. In other words, it is unclear whether the neuroprotective effects of linalool against $\mathrm{A} \beta$ toxicity were entirely attributable to $\mathrm{A} \beta$ reduction itself or whether the neuroprotective effects were also affected by antioxidant and antiinflammatory properties of linalool as well as $\mathrm{A} \beta$ reduction. However, as $\mathrm{A} \beta$ reduction was not observed in our model, our results clearly demonstrate that linalool acts as a neuroprotective agent regardless of $\mathrm{A} \beta$ levels. Similar to our results, linalool reduced apoptosis and oxidative stress and attenuated cognitive deficits in a mouse model in which $\mathrm{A} \beta$ was directly injected without $\beta$-amyloidosis [24]. Collectively, linalool is expected to inhibit $\mathrm{AD}$ progression through various physiological activities.

We found that linalool protects neurons by reducing free radical production and inflammatory responses caused by $\mathrm{A} \beta 42$, thus reducing apoptosis. However, the mechanism through which linalool acted to suppress free radical formation and the inflammatory response induced by $\mathrm{A} \beta 42$ was unknown. Linalool appeared to regulate ROS through Nrf2 and p 21 and suppress NF- $\kappa \mathrm{B}$ expression, which is increased by $\operatorname{ROS}[35,36]$. Linalool is also reported to prevent mitochondrial apoptosis by inhibiting ROS via suppressing the interaction between SIRT3 and SOD2 [37], suggesting that linalool may prevent the production of free radicals by regulating the interaction between SIRT3 and SOD2 and controlling Nrf2 expression [38]. Additionally, a study reported that linalool alleviates the lipopolysaccharide-stimulated inflammatory response through the activation of the $\mathrm{Nrf} 2 / \mathrm{HO}-1$ pathway in BV2 microglia [32]. Linalool not only enhances the expression of $\mathrm{Nrf} 2$ and $\mathrm{HO}-1$ but also suppresses the expression of inflammatory mediators such as TNF- $\alpha$, IL$1 \beta$, NO, and PGE 2 [32]. Linalool also showed an inhibitory effect on inflammation via the inhibition of NF- $\kappa$ B activity in mice with allergic asthma caused by ovalbumin exposure 


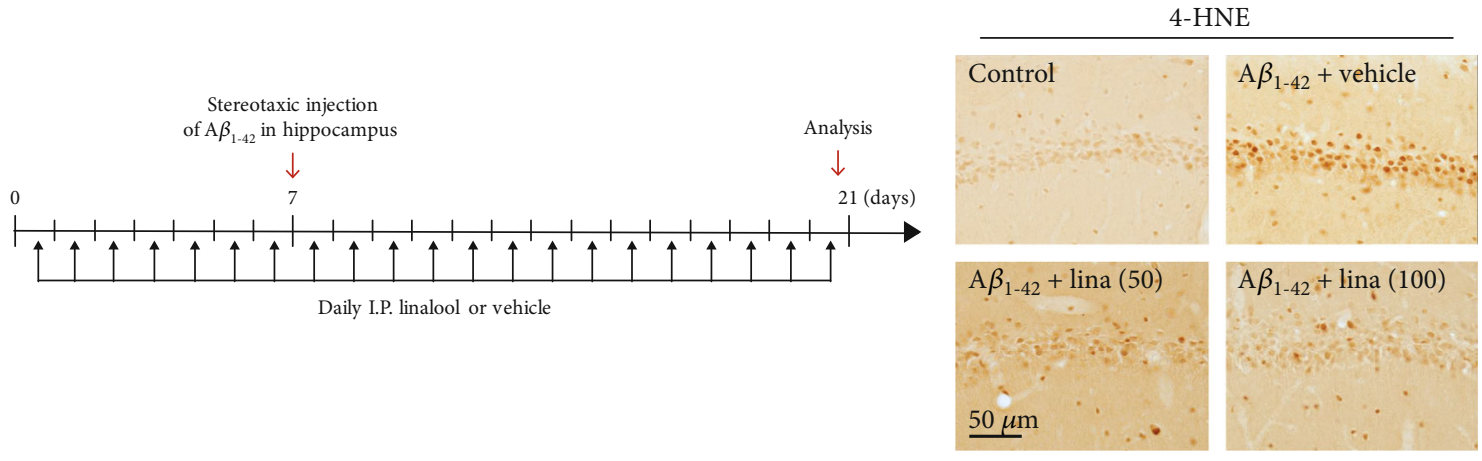

(a)

(b)
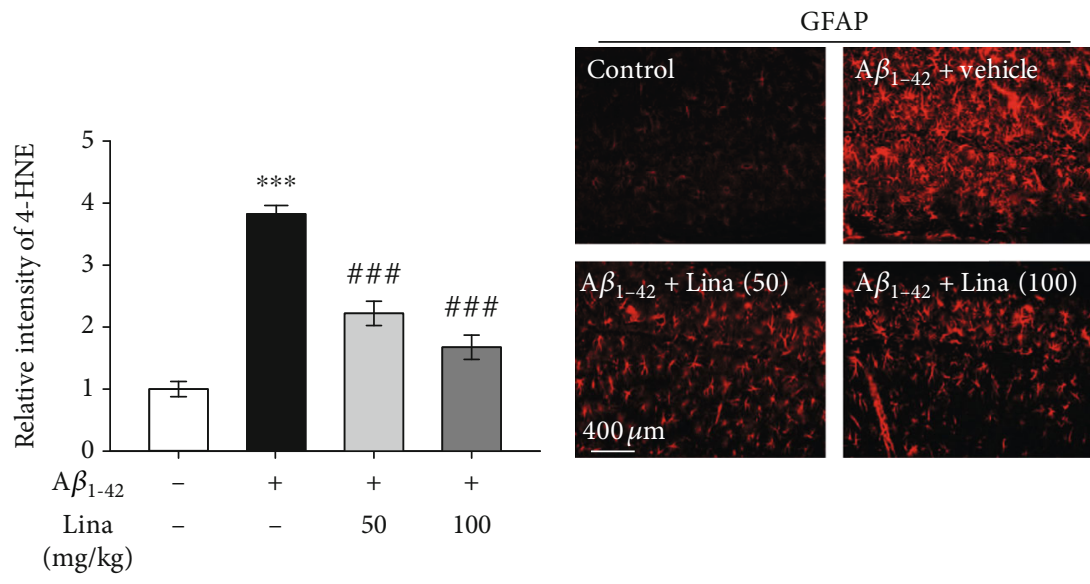

(c)
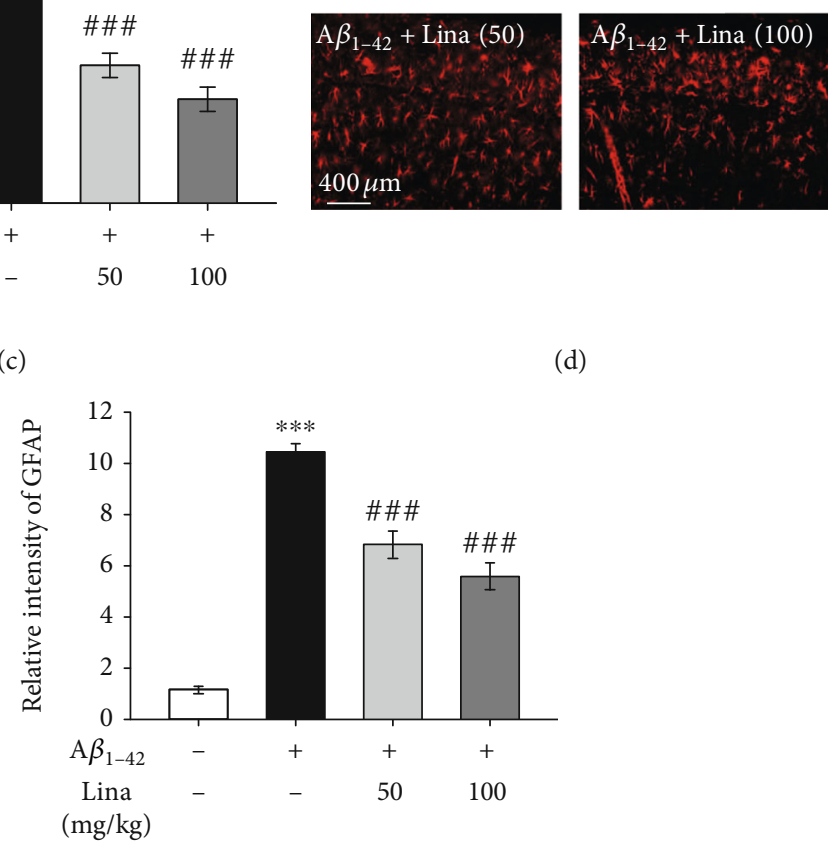

(e)

Figure 6: Linalool reduces oxidative stress and gliosis in the hippocampus of $\mathrm{A} \beta_{1-42}$-injected rats. (a) Experimental scheme. (b) Representative images of 4-HNE immunostaining, an indicator of lipid peroxidation showing oxidative stress. (c) Quantification of the intensity of 4 -HNE immunostaining in the hippocampal CA1 region $(n=5)$. Data are presented as mean \pm SEM. ${ }^{* * *} P<0.001$ vs. vehicletreated rats, ${ }^{\# \#} P<0.001$ vs. A $\beta_{1-42}$-injected rats; Tukey's multiple comparison test. (d) Representative images of GFAP immunostaining in hippocampal CA1 region. (e) Quantification of the intensity of GFAP immunostaining in the hippocampal CA1 region $(n=5)$. Data are presented as mean \pm SEM. ${ }^{* * *} P<0.001$ vs. vehicle-treated rats, ${ }^{\# \#} P<0.001$ vs. A $\beta_{1-42}$-injected rats; Tukey's multiple comparison test. I.P.: intraperitoneal injection; Lina (50): linalool $50 \mathrm{mg} / \mathrm{kg}$ I.P.; Lina (100): linalool $100 \mathrm{mg} / \mathrm{kg}$ I.P.

[39]. Future studies are warranted to confirm whether linalool reduces the $\mathrm{A} \beta 42$-stimulated inflammatory response through the regulation of the Nrf2/HO-1 pathway and NF$\kappa \mathrm{B}$ activity. Moreover, changes in the expression levels of inflammatory mediators TNF- $\alpha$, IL- $1 \beta$, and PGE 2 by linalool treatment in $\mathrm{AD}$ models should also be addressed.

$\mathrm{AD}$ is often accompanied by depression [40], and depression is reported to increase fibrous tangles in $\mathrm{AD}$ [41]. Linalool is known to have a beneficial effect on depression, which is one of the main symptoms of AD. In fact, linalool activates $\mathrm{GABA}_{\mathrm{A}}$ receptors in the brain through smell and reduces depression [42]. When the sense of smell is blocked, linalool cannot activate the $\mathrm{GABA}_{\mathrm{A}}$ receptor, preventing its antidepressant effect [42]. Further, it was confirmed that there were fewer functional $\mathrm{GABA}_{\mathrm{A}}$ receptors in the $\mathrm{AD}$ brain than in the normal brain [43]. Depression medications have no effect on depression associated with $\mathrm{AD}$, and to date, no medication is available that treats both diseases [44]. However, 
linalool can suppress depression through the $\mathrm{GABA}_{\mathrm{A}}$ receptor and $\mathrm{AD}$ progression via its antioxidant and antiinflammatory properties; thus, it may have an effect on AD-related depression.

Recently, many AD drugs have been developed, among which therapeutic agents developed by immunologic approaches targeting $\mathrm{A} \beta$ clearing have attracted attention $[45,46]$. Among them, both monoclonal antibodies and intravenous immunoglobulin showed significant results in the reduction of $\mathrm{A} \beta$ plaques in clinical trials, but the $\mathrm{AD}$ patient's cognitive improvement effect was insufficient [4750]. Therefore, in the treatment of $\mathrm{AD}$, it is possible that therapeutic agents that act on pathological mechanisms, such as inflammatory response and ROS production increased by $\mathrm{A} \beta$ in addition to removal of $\mathrm{A} \beta$, may be effective. In particular, for the treatment of multifactorial diseases such as AD, the development of therapeutic agents using natural products such as linalool, which regulates various biological targets, should be considered.

\section{Conclusions}

In conclusion, the results of this study demonstrate that linalool prevents the neurotoxicity of $\mathrm{A} \beta$ by inhibiting free radical production and inflammatory response. Therefore, linalool is expected to be used as a preventive or therapeutic agent for $\mathrm{AD}$.

\section{Data Availability}

All data generated or analyzed during this study are included in this published article.

\section{Conflicts of Interest}

The authors declare that they have no potential conflicts of interest, including any financial, personal, or other relationships, with other people or organizations.

\section{Authors' Contributions}

Chunyu Yuan and Myeongcheol Shin performed the experiments and wrote the manuscript; Youngjae Park, Byoungyun Choi, Seokhui Jang, and Chaejin Lim performed the experiments and contributed to the data analysis; Hye Sup Yun and Im-Soon Lee contributed to designing the experiment and the data analysis; and So-Yoon Won and Kyoung Sang Cho designed and supervised the study, wrote the manuscript, and read the final manuscript. Chunyu Yuan, Myeongcheol Shin, and Youngjae Park contributed equally to this work.

\section{Acknowledgments}

We thank the Bloomington Drosophila Stock Center (Bloomington, IN, USA) and Dr. Fernandez-Funez (University of Florida, USA) for providing Drosophila strains used in this study. We also would like to thank Editage (http://www .editage.co.kr) for English language editing. This work was supported by the Konkuk University Research Fund in 2017 [2017-A019-0215 (K.S.C)].

\section{References}

[1] C. Patterson, World Alzheimer Report 2018: The State of the Art of Dementia Research: New Frontiers, Alzheimer's Disease International, 2018.

[2] J. A. Hardy and G. A. Higgins, "Alzheimer's disease: the amyloid cascade hypothesis,” Science, vol. 256, no. 5054, pp. 184185, 1992.

[3] D. A. Butterfield, J. Drake, C. Pocernich, and A. Castegna, "Evidence of oxidative damage in Alzheimer's disease brain: central role for amyloid $\beta$-peptide," Trends in Molecular Medicine, vol. 7, no. 12, pp. 548-554, 2001.

[4] I. Benilova, E. Karran, and B. de Strooper, "The toxic A $\beta$ oligomer and Alzheimer's disease: an emperor in need of clothes," Nature Neuroscience, vol. 15, no. 3, pp. 349-357, 2012.

[5] M. T. Heneka, M. J. Carson, J. E. Khoury et al., "Neuroinflammation in Alzheimer's disease," The Lancet Neurology, vol. 14, no. 4, pp. 388-405, 2015.

[6] D. Selkoe and J. Hardy, "The amyloid hypothesis of Alzheimer's disease at 25 years," EMBO Molecular Medicine, vol. 8, no. 6, pp. 595-608, 2016.

[7] P. N. Tariot and H. J. Federoff, "Current treatment for Alzheimer disease and future prospects," Alzheimer Disease \& Associated Disorders, vol. 17, SUPPLEMENT 4, pp. S105-S113, 2003.

[8] R. A. Hansen, G. Gartlehner, A. P. Webb, C. G. Moore, D. E. Jonas, and D. E. Jonas, "Efficacy and safety of donepezil, galantamine, and rivastigmine for the treatment of Alzheimer's disease: a systematic review and meta-analysis," Clinical Interventions in Aging, vol. 3, no. 2, pp. 211-225, 2008.

[9] L. G. Howes, "Cardiovascular effects of drugs used to treat Alzheimer's disease," Drug Safety, vol. 37, no. 6, pp. 391-395, 2014.

[10] A. Aharoni, M. Jongsma, and H. Bouwmeester, "Volatile science? Metabolic engineering of terpenoids in plants," Trends in Plant Science, vol. 10, no. 12, pp. 594-602, 2005.

[11] F. Loreto, F. Bagnoli, and S. Fineschi, "One species, many terpenes: matching chemical and biological diversity," Trends in Plant Science, vol. 14, no. 8, pp. 416-420, 2009.

[12] K. S. Cho, Y. R. Lim, K. Lee, J. Lee, J. H. Lee, and I. S. Lee, “Terpenes from forests and human health," Toxicological Research, vol. 33, no. 2, pp. 97-106, 2017.

[13] M. Shin, Q. F. Liu, B. Choi et al., "Neuroprotective effects of limonene (+) against A $\beta 42$-induced neurotoxicity in a Drosophila model of Alzheimer's disease," Biological and Pharmaceutical Bulletin, vol. 43, no. 3, pp. 409-417, 2020.

[14] C. S. Letizia, J. Cocchiara, J. Lalko, and A. Api, "Fragrance material review on linalool," Food and Chemical Toxicology, vol. 41, no. 7, pp. 943-964, 2003.

[15] I. Pereira, P. Severino, A. C. Santos, E. B. Souto, and E. B. Souto, "Linalool bioactive properties and potential applicability in drug delivery systems," Colloids and Surfaces B: Biointerfaces, vol. 171, pp. 566-578, 2018.

[16] H. Casabianca, J. Graff, V. Faugier, F. Fleig, and C. Grenier, "Enantiomeric distribution studies of linalool and linalyl acetate. A powerful tool for authenticity control of essential oils," Journal of High Resolution Chromatography, vol. 21, no. 2, pp. 107-112, 1998.

[17] A. C. Aprotosoaie, M. Hăncianu, I. I. Costache, and A. Miron, "Linalool: a review on a key odorant molecule with valuable 
biological properties," Flavour and Fragrance Journal, vol. 29, no. 4, pp. 193-219, 2014.

[18] A. T. Peana, S. Marzocco, A. Popolo, and A. Pinto, “(-)-linalool inhibits in vitro NO formation: probable involvement in the antinociceptive activity of this monoterpene compound," Life Science, vol. 78, no. 7, pp. 719-723, 2006.

[19] R. Kannappan, S. C. Gupta, J. H. Kim, B. B. Aggarwal, and B. B. Aggarwal, "Neuroprotection by spice-derived nutraceuticals: you are what you eat!," Molecular Neurobiology, vol. 44, no. 2, pp. 142-159, 2011.

[20] S. Mehri, M. A. Meshki, and H. Hosseinzadeh, "Linalool as a neuroprotective agent against acrylamide-induced neurotoxicity in Wistar rats," Drug and Chemical Toxicology, vol. 38, no. 2, pp. 162-166, 2015.

[21] G. H. Seol, P. Kang, H. S. Lee, and G. H. Seol, "Antioxidant activity of linalool in patients with carpal tunnel syndrome," BMC Neurology, vol. 16, no. 1, p. 17, 2016.

[22] A. Lapczynski, C. S. Letizia, and A. M. Api, "Fragrance material review on d-linalool," Food and Chemical Toxicology, vol. 46, no. 11, pp. S193-S194, 2008.

[23] A. M. Sabogal-Guáqueta, E. Osorio, and G. P. CardonaGómez, "Linalool reverses neuropathological and behavioral impairments in old triple transgenic Alzheimer's mice," Neuropharmacology, vol. 102, pp. 111-120, 2016.

[24] P. Xu, K. Wang, C. Lu et al., "Protective effects of linalool against amyloid beta-induced cognitive deficits and damages in mice," Life Science, vol. 174, pp. 21-27, 2017.

[25] K. Iijima and K. Iijima-Ando, "Drosophila models of Alzheimer's amyloidosis: the challenge of dissecting the complex mechanisms of toxicity of amyloid- $\beta 42$," Journal of Alzheimer's Disease, vol. 15, no. 4, pp. 523-540, 2008.

[26] J. K. Ryu, S. Franciosi, P. Sattayaprasert, S. U. Kim, and J. G. McLarnon, "Minocycline inhibits neuronal death and glial activation induced by beta-amyloid peptide in rat hippocampus," Glia, vol. 48, no. 1, pp. 85-90, 2004.

[27] G. Paxinos and C. Watson, The rat brain in sterotaxic coordinates, Academic Press, San Diego, 1998.

[28] M. Haddadi, S. R. Jahromi, B. K. C. Sagar, T. Shivanandappa, S. R. Ramesh, and S. R. Ramesh, "Brain aging, memory impairment and oxidative stress: A study in Drosophila melanogaster," Behavioural Brain Research, vol. 259, pp. 60-69, 2014.

[29] B. Urbanc, L. Cruz, R. le et al., "Neurotoxic effects of thioflavin S-positive amyloid deposits in transgenic mice and Alzheimer's disease," Proceedings of the National Academy of Sciences of the United States of America, vol. 99, no. 22, pp. 13990-13995, 2002.

[30] H. Kadowaki, H. Nishitoh, F. Urano et al., "Amyloid_ $\beta$ _induces neuronal cell death through ROS-mediated ASK1 activation," Cell Death \& Differentiation, vol. 12, no. 1, pp. 19-24, 2005.

[31] M. Huo, X. Cui, J. Xue et al., “Anti-inflammatory effects of linalool in RAW 264.7 macrophages and lipopolysaccharideinduced lung injury model," Journal of Surgical Research, vol. 180, no. 1, pp. e47-e54, 2013.

[32] Y. Li, O. Lv, F. Zhou, Z. Wu, Y. Zheng, and Y. Zheng, "Linalool inhibits LPS-induced inflammation in BV2 microglia cells by activating Nrf2," Neurochemical Research, vol. 40, no. 7, pp. 1520-1525, 2015.

[33] K. Liu, Q. Chen, Y. Liu, X. Wang, and X. Wang, "Isolation and biological activities of decanal, linalool, valencene, and octanal from sweet orange oil," Journal of Food Science, vol. 77, no. 11, pp. C1156-C1161, 2012.
[34] H. Park, G. H. Seol, S. Ryu, and I. Y. Choi, "Neuroprotective effects of (-)-linalool against oxygen-glucose deprivationinduced neuronal injury," Archives of Pharmacal Research, vol. 39, no. 4, pp. 555-564, 2016.

[35] I. O. Tekeli, A. Ateşşahin, F. Sakin, A. Aslan, S. Çeribaşı, and M. Yipel, "Protective effects of conventional and colontargeted lycopene and linalool on ulcerative colitis induced by acetic acid in rats," Inflammopharmacology, vol. 27, no. 2, pp. 313-322, 2019.

[36] S. Jana, K. Patra, S. Sarkar et al., "Antitumorigenic potential of linalool is accompanied by modulation of oxidative stress: an in vivo study in sarcoma-180 solid tumor model," Nutrition and Cancer, vol. 66, no. 5, pp. 835-848, 2014.

[37] H. Kim, Y. D. Lee, H. J. Kim, H. H. Kim, and H. H. Kim, "SOD2 and Sirt3 control osteoclastogenesis by regulating mitochondrial ROS," Bone and Mineral Research, vol. 32, no. 2, pp. 397-406, 2017.

[38] Y. Cheng, C. Dai, and J. Zhang, "SIRT3-SOD2-ROS pathway is involved in linalool-induced glioma cell apoptotic death," Acta Biochimica Polonica, vol. 64, no. 2, pp. 343-350, 2017.

[39] M. G. Kim, S. M. Kim, J. H. Min et al., "Anti-inflammatory effects of linalool on ovalbumin-induced pulmonary inflammation," International Immunopharmacology, vol. 74, article 105706, 2019.

[40] R. E. Wragg and D. V. Jeste, "Overview of depression and psychosis in Alzheimer's disease," The American Journal of Psychiatry, vol. 146, no. 5, pp. 577-587, 1989.

[41] M. A. Rapp, M. Schnaider-Beeri, H. T. Grossman et al., "Increased hippocampal plaques and tangles in patients with Alzheimer disease with a lifetime history of major depression," Archives of General Psychiatry, vol. 63, no. 2, pp. 161-167, 2006.

[42] H. Harada, H. Kashiwadani, Y. Kanmura, and T. Kuwaki, "Linalool odor-induced anxiolytic effects in mice," Frontiers in Behavioral Neuroscience, vol. 12, p. 241, 2018.

[43] R. A. Rissman, A. L. de Blas, and D. M. Armstrong, "GABAAreceptors in aging and Alzheimer's disease," Journal of Neurochemistry, vol. 103, no. 4, pp. 1285-1292, 2007.

[44] T. Cassano, S. Calcagnini, A. Carbone et al., "Pharmacological treatment of depression in Alzheimer's disease: a challenging task," Frontiers in Pharmacology, vol. 10, article 1067, 2019.

[45] F. Panza, M. Lozupone, G. Logroscino, and B. P. Imbimbo, “A critical appraisal of amyloid- $\beta$-targeting therapies for Alzheimer disease," Nature Reviews Neurology, vol. 15, no. 2, pp. 73-88, 2019.

[46] J. Delrieu, P. J. Ousset, C. Caillaud, and B. Vellas, "Clinical trials of intravenous immunoglobulin for Alzheimer's disease," Journal of Clinical Immunology, vol. 120, no. 1, pp. 186-193, 2012.

[47] K. G. Yiannopoulou, A. I. Anastasiou, V. Zachariou, and S. H. Pelidou, "Reasons for failed trials of disease-modifying treatments for Alzheimer disease and their contribution in recent research," Biomedicines, vol. 7, no. 4, p. 97, 2019.

[48] R. Dodel, F. Neff, C. Noelker et al., "Intravenous immunoglobulins as a treatment for Alzheimer's disease," Drugs, vol. 70, no. 5, pp. 513-528, 2010.

[49] N. D. Prins and P. Scheltens, “Treating Alzheimer's disease with monoclonal antibodies: current status and outlook for the future," Alzheimer's Research \& Therapy, vol. 5, no. 6, p. 56, 2013.

[50] N. Relkin, "Clinical trials of intravenous immunoglobulin for Alzheimer's disease," Journal of Clinical Immunology, vol. 34, no. 1, pp. S74-S79, 2014. 